\title{
Quantum transport in Sierpinski carpets
}

\author{
Edo van Veen, ${ }^{1}$ Shengjun Yuan,,${ }^{1, *}$ Mikhail I. Katsnelson, ${ }^{1}$ Marco Polini,${ }^{2}$ and Andrea Tomadin ${ }^{3}$ \\ ${ }^{1}$ Institute for Molecules and Materials, Radboud University, Heyendaalseweg 135, 6525AJ Nijmegen, The Netherlands \\ ${ }^{2}$ Istituto Italiano di Tecnologia, Graphene Labs, Via Morego 30, I-16163 Genova, Italy \\ ${ }^{3}$ NEST, Istituto Nanoscienze-CNR and Scuola Normale Superiore, I-56126 Pisa, Italy
}

(Received 15 April 2015; revised manuscript received 26 January 2016; published 21 March 2016)

\begin{abstract}
Recent progress in the design and fabrication of artificial two-dimensional (2D) materials paves the way for the experimental realization of electron systems moving on complex geometries, such as plane fractals. In this work, we calculate the quantum conductance of a 2D electron gas roaming on a Sierpinski carpet (SC), i.e., a plane fractal with Hausdorff dimension intermediate between 1 and 2 . We find that the fluctuations of the quantum conductance are a function of energy with a fractal graph, whose dimension can be chosen by changing the geometry of the SC. This behavior is independent of the underlying lattice geometry.
\end{abstract}

DOI: 10.1103/PhysRevB.93.115428

A variety of experimental protocols that can be used to create artificial two-dimensional (2D) periodic lattices for electrons, atoms, and photons is available nowadays. For example, schemes for creating artificial hexagonal lattices [1] allowed to observe a wealth of interesting phenomena, such as Mott-Hubbard split bands [2], massless Dirac fermion behavior modified by pseudoelectric and pseudomagnetic fields [3], and photonic Floquet topological insulating states [4]. In the case of ultracold atomic gases loaded in hexagonal optical lattices, recent progress has even led to the experimental realization [5] of the Haldane model [6].

More generally, in the context of solid-state implementations, a combination of $e$-beam nanolithography, etching, and metallic gate deposition [7-13] can in principle yield high-quality two-dimensional (2D) patterns with arbitrary, nonperiodic shape in semiconductor heterostructures (such as GaAs/AlGaAs) hosting ultrahigh-mobility 2D electron gases (EGs). Ultimately, these procedures yield an external potential landscape with the desired geometry that acts as a potential well to trap electrons. Synthetic solid-state quantum materials can also be created by utilizing scanning probe methods [3].

Further improvements in spatial resolution can be obtained by bottom-up nanofabrication methods such as nanocrystal self-assembly [14]. Both the local electronic structure [15] and the geometry of the system [16] can be designed by careful choices of precursor molecules and reaction parameters. In particular, usage of building blocks with chiral bondings on a substrate with a compatible symmetry allowed the assembly of molecular Sierpinski triangle fractals [16]. These experimental achievements motivate the theoretical investigation of complex $2 \mathrm{D}$ structures, with the aim of discovering novel transport and optical features which could enable or improve technological applications.

In this paper, we present a theoretical study of the transport properties of a 2DEG in a Sierpinski carpet (SC), which is a 2D self-similar structure [17] shown in Fig. 1(a). Macroscopically, the self-similarity of the SC is quantified by the fact that its Hausdorff dimension [17] $d_{\mathrm{H}}$ (i.e., a generalization of the topological dimension) is between 1 (a line) and 2 (a

\footnotetext{
*s.yuan@science.ru.nl
}

plane), which makes the SC a fractal [18]. By varying the parameters $\mathcal{N}$ and $\mathcal{L}$ of the geometrical construction of the fractal, defined in the caption of Fig. 1, a family of SCs with different dimensions can be generated. At a microscopic level, any physical realization of a $2 \mathrm{DEG}$ in a SC will involve electrons hopping on a lattice. Here, we have considered three different underlying lattice structures, i.e. triangular, square, and hexagonal lattices; see Figs. 1(b)-1(d). The last case is particularly relevant, since it models a top-down nanofabricated SC obtained by etching a graphene sheet.

In contrast to electrons hopping on Bloch translationally invariant lattices, the SC hosts both extended-Fig. 1(e)and localized-Fig. 1(f) - electronic states in narrow energy ranges. Phase-coherent electronic transport through the SC, thus, depends noticeably on the carrier energy and on the geometric matching between lead positions and profiles of the extended electronic states. The quantum conductance can reach the maximum value allowed by the number of open channels in the leads [19], depending on the lead positions and their widths, and displays fractal fluctuations [21-25] as a function of energy, in the absence of a magnetic field. At odds with the vast majority of the literature on fractal conductance fluctuations (CFs), which considers geometrically simple structures such as billiards, here we find that, in a SC, the fractal dimension of the sample determines the fractal dimension of the CFs [26].

We model a 2DEG in a SC by means of a single-orbital tight-binding Hamiltonian:

$$
\hat{\mathcal{H}}=-t \sum_{\langle i, j\rangle, \sigma}\left(\hat{c}_{i, \sigma}^{\dagger} \hat{c}_{j, \sigma}+\text { H.c. }\right) .
$$

This Hamiltonian describes electrons with spin $\sigma=\uparrow, \downarrow$ hopping between the nearest-neighbor sites $\langle i, j\rangle$ of a SC. Nanopattering a SC on the surface of a semiconductor hosting a high mobility 2DEG is expected $[2,7,8]$ to yield $t$ of the order of a few meV, while protocols based on scanning tunneling microscope (STM) manipulation [3] are expected to yield $t \sim 100 \mathrm{meV}$. For the sake of simplicity, we ignore magnetic fields and electron-electron interactions, which are expected to lead to very interesting quantitative and qualitative effects. Conductance and wave function calculations are performed by using KWANT [41]. In this toolkit, wave function 


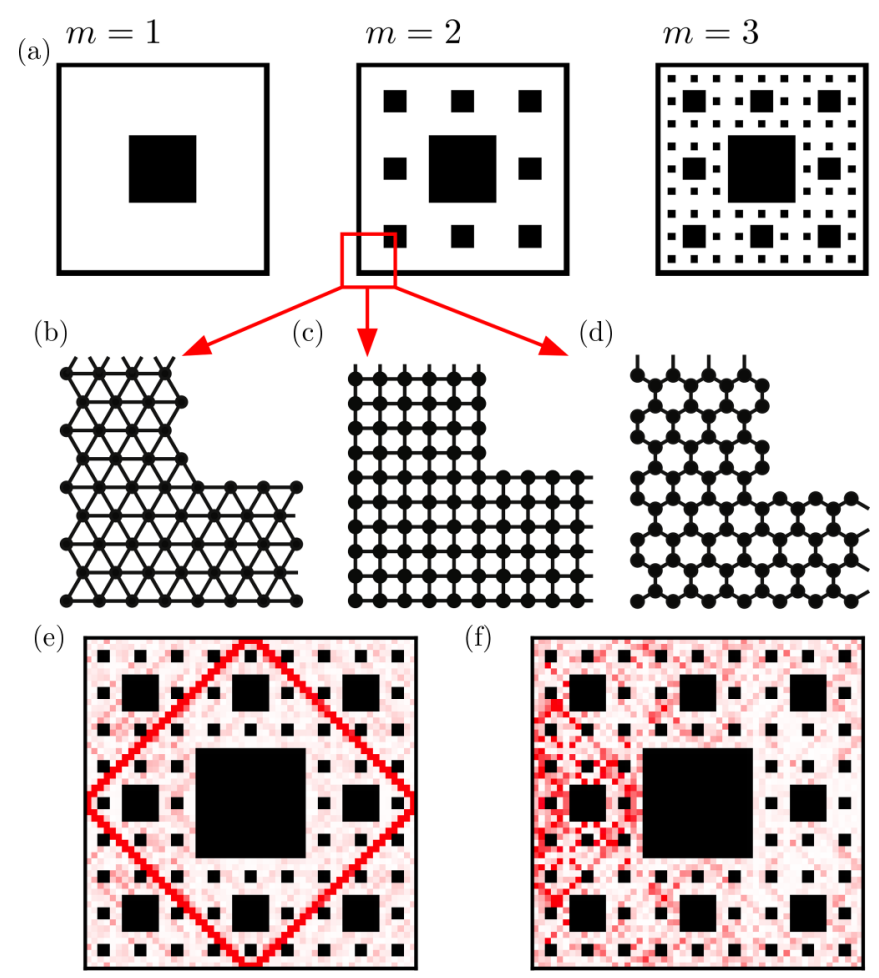

FIG. 1. (a) Top-down geometric construction of a Sierpinski carpet (SC). The black squares represent regions that are removed from the white sample. At the $m$ th iteration one removes $\mathcal{N}$ copies of the regions removed at the $(m-1)$ th iteration, after scaling them down in linear size by a factor $\mathcal{L}$. [In (a), $\mathcal{N}=8$ and $\mathcal{L}=3$.] For a number of iterations $m \gg 1$ one obtains an approximation of the SC, a plane fractal with dimension $d_{\mathrm{H}}=\log _{\mathcal{L}} \mathcal{N}[\simeq 1.89$ in (a)]. (b)-(d) The triangular, square, and hexagonal underlying lattices considered in this work. The width of the sample is $n$ lattice cells. (e) and (f) Examples of delocalized and localized electronic states in a SC with an underlying square lattice. The square modulus $\left|\psi\left(\boldsymbol{r}_{i}\right)\right|^{2}$ of the electron wave function is shown, with a color scale varying from white (zero) to red (maximum). Because of the fractal geometry of the sample, delocalized and localized states coexist in narrow energy ranges, producing fractal conductance fluctuations (see Fig. 2).

matching is implemented to compute the wave functions in the scattering region and the scattering matrix $S_{i j}$ for an incoming propagating mode $i$ and an outgoing mode $j$. The conductance $G$ between the left lead $\mathrm{L}$ and the right lead $\mathrm{R}$ is given by the Landauer formula

$$
G=\frac{2 e^{2}}{h} \sum_{i \in \mathrm{L}, j \in \mathrm{R}}\left|S_{i j}\right|^{2} .
$$

With reference to Fig. 1, each discretized SC is characterized by the iteration step $m$, its underlying lattice structure (triangular, square, and hexagonal), and the total number of sites $n$ along the bottom edge.

We have calculated the energy dependence of the twoterminal conductance $G(E)$ of the tight-binding model (1) for a configuration with "central" leads (i.e., a configuration in which two leads are attached to the center of the left and right sides of the SC [20]) and one with "diagonal" leads (i.e. leads attached to the bottom of the left side and to the top of the
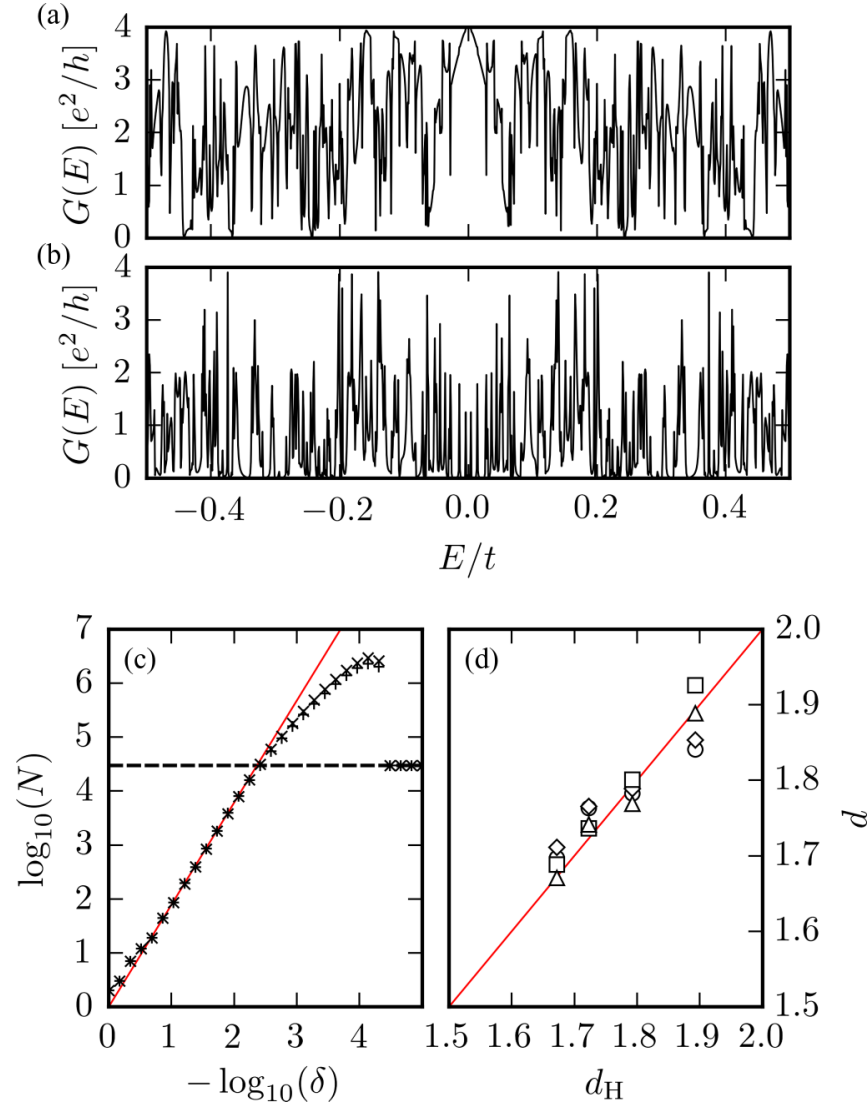

FIG. 2. (a) and (b) Energy dependence of the conductance $G(E)$ (in units of $e^{2} / h$ ) of a square-lattice SC with $\mathcal{N}=8, \mathcal{L}=3, m=3$, and $n=54$. Data in (a) and (b) refer to central and diagonal lead positions, respectively. (c) $\mathrm{BC}$ algorithm analysis of the conductance fluctuations for SCs with geometry as in (a) (+) and (b) $(\times)$, for $m=$ 4 , and $n=162$. The horizontal dashed lines represent the saturation value $N=N_{\mathrm{s}}$, with $N_{\mathrm{s}}=3 \times 10^{4}$. The slope $d$ of the solid line has been set equal to the Hausdorff dimension $d_{\mathrm{H}} \simeq 1.89$ of the SC. (d) BC dimension $d$ of the conductance fluctuations for square-lattice SCs with different dimensions for $m=3, n=54(\circ, \diamond)$, and $m=4$, $n=162(\square, \triangle)$. Results for both center $(\circ, \square)$ and diagonal $(\diamond, \triangle)$ lead positions are shown. The solid line represents $d=d_{\mathrm{H}}$.

right side of the SC [20]). We first focus on the geometrically simpler case of the square lattice (Fig. 2) and then present results on the triangular and hexagonal lattices (Fig. 3).

The quantum conductance $G$ of a square-lattice $\mathrm{SC}$ as a function of energy $E$ is shown in Figs. 2(a) and 2(b). (We show an energy range smaller than the whole bandwidth to distinguish the single peaks in the profile.) In a physical realization of a $2 \mathrm{DEG}$, the energy $E$ is determined by the gate potential. In Fig. 2(a) we clearly see that the two-terminal conductance $G(E)$ is equal to $4 e^{2} / h$ for $E=0$, where a conductive extended state is present [29]. This is because, with central lead positions, electrons of a given spin injected on the left side of the SC can reach the right side by following two equivalent paths, Fig. 1(e), each carrying a conductance quantum, without being backscattered by the inner holes of the SC. On the other hand, as we can see from Fig. 2(b), the $\mathrm{SC}$ can be insulating (i.e., $G=0$ ) at the same energy, when probed with leads in a diagonal configuration, which do not 

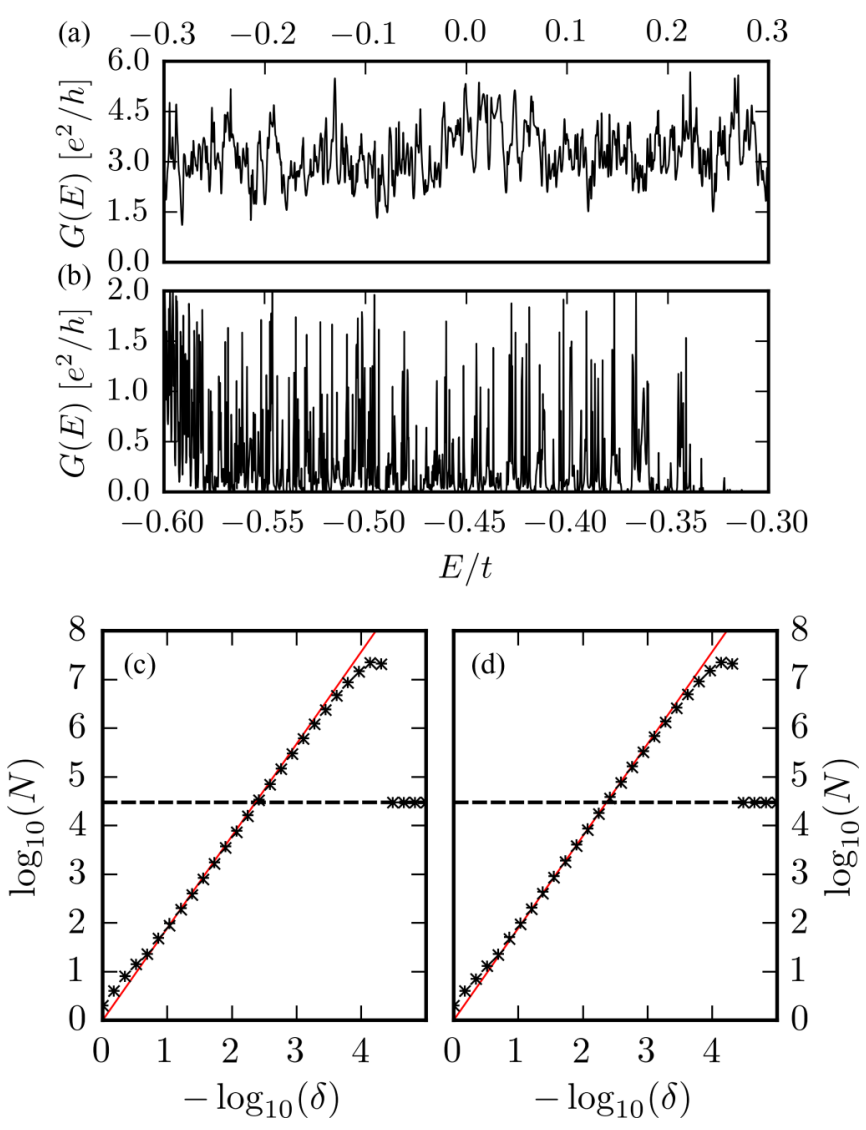

FIG. 3. (a) and (b) Energy dependence of the conductance $G(E)$ (in units of $e^{2} / h$ ) of a SC with $\mathcal{N}=8, \mathcal{L}=3, m=3$, and central lead positions. Data in (a) refer to a triangular lattice with $n=101$, while data in (b) refer to a hexagonal lattice with $n=101$. (c) BC algorithm analysis for a triangular-lattice SC with $\mathcal{N}=8, \mathcal{L}=3, m=4$, and $n=284$. (d) BC algorithm analysis for a hexagonal-lattice SC with $\mathcal{N}=8, \mathcal{L}=3, m=4$, and $n=284$. Results for both center (+) and diagonal $(\times)$ leads are shown in (c) and (d). The slope $d$ of the solid line has been set equal to the dimension $d_{\mathrm{H}} \simeq 1.89$ of the SC.

couple to the conducting state. The robustness of these features to different types of (localized or smoothly varying) disorder is discussed in Ref. [20].

The roughness of the $\mathrm{CF}$ graph increases with the iteration step $m$ of the construction of the SC. As $m$ is increased, finer and finer CFs appear, at progressively smaller energy scales. This suggests that the CF graph is actually a fractal, with a dimension larger than its topological dimension (1). The dimension of the $\mathrm{CF}$ graph can be quantified by using a box-counting (BC) algorithm [23]. This algorithm counts the number $N$ of squares of size $\delta$ which are necessary to continuously cover the graph of $G(E)$ (in units of $e^{2} / h$ ) rescaled to a unit square. In general, points in the plane $(\log N-\log \delta)$ are expected to fall in three distinct regions. For large values of $\delta$, the squares are too large to distinguish the features of the graph and $N$ grows slowly as $\delta$ decreases. For very small values of $\delta$, the squares are so small that they resolve the single points in the set of data belonging to the $\mathrm{CF}$ graph: in this case $N$ is expected to saturate to the number $N_{\mathrm{s}}$ of points in the energy mesh where $G(E)$ is evaluated. Finally, there is an intermediate region (usually called the "scaling region") where scaling is linear in a log-log plot, i.e., where $N \sim \delta^{-d}$. The slope $d$ in the scaling region is the BC estimate of the dimension of the CF [20].

In Figs. 2(c) and 2(d), we show the results of the BC algorithm for the CFs of a square-lattice $\mathrm{SC}$ with $\mathcal{N}=8$ and $\mathcal{L}=3$, as in Fig. 1(a). The analyzed CFs clearly show a fractal dimension $1<d<2$ over a scaling region of more than two orders of magnitude. The fractal nature of the CF graph stems from the coexistence of extended and localized electron wave functions in narrow energy ranges [21,23]. We stress that localized electron wave functions emerge in our SC, and even in the much simpler Sierpinski gasket [37] (see below), in the absence of elastic disorder, because of scattering of electrons against the inner holes of the SC. Most importantly, we find that the results of the $\mathrm{BC}$ algorithm are independent of the lead positions. This allows us to claim that the dimension of the $\mathrm{CF}$ graph is an intrinsic property of the sample geometry.

In Fig. 2(d), we show the $\mathrm{BC}$ algorithm estimate of the fractal dimension $d$ for SCs with different dimensions, obtained by changing $\mathcal{N}$ and $\mathcal{L}$ in the iterative construction illustrated in Fig. 1(a). BC algorithm analyses for $m=3$ or $m=4$, or for different lead configurations, yield values of $d$ which differ by a few percent. However, $d$ substantially depends on the Hausdorff dimension $d_{\mathrm{H}}$ of the SC. A reasonable conjecture, supported by our numerical results, is that $d=d_{\mathrm{H}}$ for $m \gg 1$. It is remarkable that the analysis of CFs carries information on the SC geometry, down to very small length scales. Conversely, these results show that it is possible to fix the fluctuation spectrum of the quantum conductance by choosing an appropriate SC. This evidence that the fractal dimension of the sample determines the fractal dimension of the $\mathrm{CF}$ graph is the main result of this paper.

In Fig. 3, we test the generality of our findings by extending our numerical analysis to SCs with underlying triangular and hexagonal lattices. From Figs. 3(a) and 3(b) we clearly see that the conductance graphs of triangular- and hexagonal-lattice SCs are strikingly different from each other and from the conductance graph of square-lattice SCs. It is remarkable that, notwithstanding the different appearances, the CF graph in all three cases yield very similar BC algorithm results. In particular, the estimated BC dimension of triangular- and hexagonal-lattice SCs is compatible with our conjecture $d=$ $d_{\mathrm{H}}$.

In passing, we notice that a gap appears in the conductance spectrum $G(E)$ of the hexagonal-lattice $\mathrm{SC}$, where the conductance exactly vanishes. In Fig. 3(b) we show the conductance on an energy range below the gap. We point out, however, that the corresponding density of states of the hexagonal-lattice SC [20] does not display a gap.

Finally, to shed further light on the origin of the fractal CFs, we calculate the quantum conductance of two fractals which do not belong to the family of SCs. In panel (a) of Fig. 4 we show an example of a Sierpinski gasket [37], while in panel (b) we report a Vicsek fractal [42]. For simplicity, we limited our investigation to plane fractals which can be obtained iteratively, as discussed in the caption of Fig. 1. In the case of a gasket, one starts from a triangle, while for the Vicsek fractal one starts from a square cross. The BC algorithm analysis applied to the CFs of the gasket and Vicsek fractal is shown in panels (c) and (d). We see that both geometries feature 

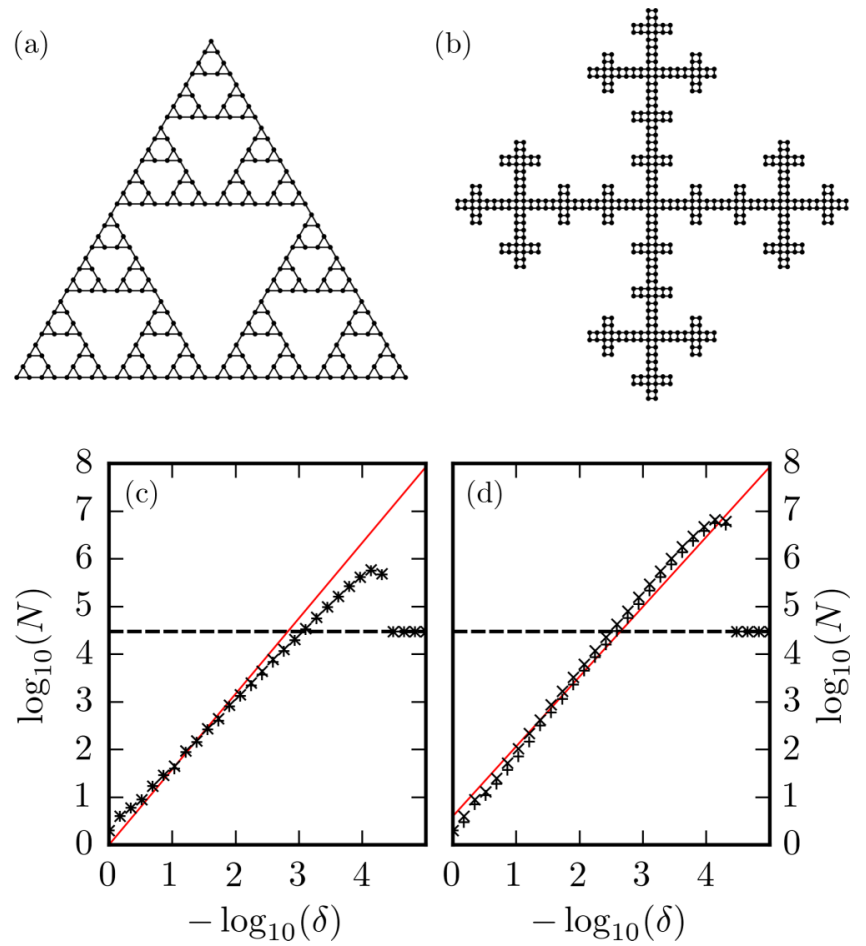

FIG. 4. (a) A Sierpinski gasket $(\mathcal{N}=3, \mathcal{L}=2)$, obtained with $m=5$ iteration steps, with $n=32$ lattice sites along the base. (b) A Vicsek fractal $(\mathcal{N}=5, \mathcal{L}=3)$, obtained with $m=3$ iteration steps, with $n=54$ lattice sites along the main cross arm. (c) and (d) BC analysis for a gasket with $m=8$ and $n=256$ and a Vicsek fractal with $m=6$ and $n=1458$. The slope of the solid line has been set equal to the Hausdorff dimension of the samples. Results for two different lead locations are shown with different symbols in (c) and (d).

fractal CFs, but the difference between $d$ and $d_{\mathrm{H}}$ in the case of the gasket and Vicsek fractal is sizable contrary to the case of a SC [20]. For increasing $m$, the box counting dimension of the conductance fluctuations of the gasket converges to $d=1.22$ $\left(d_{\mathrm{H}}=1.58\right)$, and for the Vicsek fractal to $d=1.69\left(d_{\mathrm{H}}=\right.$ 1.46). Moreover, the gasket and Vicsek fractals show multiple extended regions where the conductance is zero, whereas for the carpets the conductance fluctuates heavily over the entire energy range, except around $E=0$ for the hexagonal-lattice case.

A qualitative difference between the SC and the gasket/Vicsek fractal is the value of their "ramification number" [43], i.e., the number of bonds that must be cut in order to isolate different iterations of the lattice. For the gasket and Vicsek fractal, the ramification is finite, while for the SC it is infinite. For fractals with finite ramification it is possible to give analytical solutions to the Schrödinger equation [37], while general fractals with infinite ramification are not amenable to analytical treatments. The relation between the dimensions of the samples in the SC family and of the corresponding $\mathrm{CFs}$ is possibly a consequence of the infinite ramification of the SC. Intuitively, electrons in a SC explore a larger portion of the available phase space, and thus their conduction properties are more closely related to those of the sample. Although more work is necessary to establish this connection at a formal level, we believe that our results motivate careful transport studies of planar fractal devices, which are just being made available by recent progress in nanofabrication [13] and synthetic chemistry [16].

We wish to thank Luigi Ambrosio, Raffaella Burioni, Fabio Taddei, and Sandro Wimberger for useful discussions, and Carlo Beenakker for very useful correspondence. This work was supported by the European Research Council Advanced Grant program (Contract No. 338957) (S.Y. and M.I.K.) and by the Italian Ministry of Education, University, and Research (MIUR) through the program "Progetti Premiali 2012"-Project ABNANOTECH (A.T. and M.P.). Support by the Netherlands National Computing Facilities foundation (NCF), with funding from the Netherlands Organisation for Scientific Research (NWO), is gratefully acknowledged.
[1] M. Polini, F. Guinea, M. Lewenstein, H. C. Manoharan, and V. Pellegrini, Nat. Nanotech. 8, 625 (2013).

[2] A. Singha, M. Gibertini, B. Karmakar, S. Yuan, M. Polini, G. Vignale, M. I. Katsnelson, A. Pinczuk, L. N. Pfeiffer, K. W. West, and V. Pellegrini, Science 332, 1176 (2011).

[3] K. K. Gomes, W. Mar, W. Ko, F. Guinea, and H. C. Manoharan, Nature (London) 483, 306 (2012).

[4] M. C. Rechtsman, J. M. Zeuner, Y. Plotnik, Y. Lumer, D. Podolsky, F. Dreisow, S. Nolte, M. Segev, and A. Szameit, Nature (London) 496, 196 (2013).

[5] G. Jotzu, M. Messer, R. Desbuquois, M. Lebrat, T. Uehlinger, D. Greif, and T. Esslinger, Nature (London) 515, 237 (2014).

[6] F. D. M. Haldane, Phys. Rev. Lett. 61, 2015 (1988).

[7] M. Gibertini, A. Singha, V. Pellegrini, M. Polini, G. Vignale, A. Pinczuk, L. N. Pfeiffer, and K. W. West, Phys. Rev. B 79, 241406(R) (2009).
[8] G. De Simoni, A. Singha, M. Gibertini, B. Karmakar, M. Polini, V. Piazza, L. N. Pfeiffer, K. W. West, F. Beltram, and V. Pellegrini, Appl. Phys. Lett. 97, 132113 (2010).

[9] C.-H. Park and S. G. Louie, Nano Lett. 9, 1793 (2009).

[10] E. Räsänen, C. A. Rozzi, S. Pittalis, and G. Vignale, Phys. Rev. Lett. 108, 246803 (2012).

[11] L. Nádvorník, M. Orlita, N. A. Goncharuk, L. Smrčka, V. Novák, V. Jurka, K. Hruška, Z. Výborný, Z. R. Wasilewski, M. Potemski, and K. Výborný, New J. Phys. 14, 053002 (2012).

[12] S. Goswami, M. A. Aamir, C. Siegert, M. Pepper, I. Farrer, D. A. Ritchie, and A. Ghosh, Phys. Rev. B 85, 075427 (2012).

[13] D. Scarabelli, S. Wang, Y. Y. Kuznetsova, L. N. Pfeiffer, K. West, G. C. Gardner, M. J. Manfra, V. Pellegrini, A. Pinczuk, and S. J. Wind, J. Vac. Sci. Technol. B 33, 06FG03 (2015).

[14] W. H. Evers, B. Goris, S. Bals, M. Casavola, J. de Graaf, R. van Roij, M. Dijkstra, and D. Vanmaekelberghet, Nano Lett. 13, 2317 (2013). 
[15] Y.-C. Chen, T. Cao, C. Chen, Z. Pedramrazi, D. Haberer, D. G. de Oteyza, F. R. Fischer, S. G. Louie, and M. F. Crommie, Nat. Nanotech. 10, 156 (2015).

[16] J. Shang, Y. Wang, M. Chen, J. Dai, X. Zhou, J. Kuttner, G. Hilt, X. Shao, J. M. Gottfried, and K. Wu, Nat. Chem. 7, 389 (2015).

[17] K. J. Falconer, The Geometry of Fractal Sets (Cambridge University Press, Cambridge, 1985).

[18] B. B. Mandelbrot, The Fractal Geometry of Nature (W. H. Freemand and Company, New York, 1983).

[19] Surprinsingly, we find that extended states, which are responsible for large conductance values, are quite robust to elastic disorder. See Ref. [20] online.

[20] See Supplemental Material http://link.aps.org/supplemental/ 10.1103/PhysRevB.93.115428 for details on the geometry of the fractal samples, on the calculation of the fractal dimension, and on the effects of disorder.

[21] R. Ketzmeric, Phys. Rev. B 54, 10841 (1996).

[22] A. S. Sachrajda, R. Ketzmerick, C. Gould, Y. Feng, P. J. Kelly, A. Delage, and Z. Wasilewski, Phys. Rev. Lett. 80, 1948 (1998).

[23] I. Guarneri and M. Terraneo, Phys. Rev. E 65, 015203(R) (2001).

[24] R. Taylor, R. Newbury, A. Micolich, M. Fromhold, H. Linke, G. Davies, J. Bird, T. Martin, and C. Marlow, in Electron Transport in Quantum Dots, edited by J. P. Bird (Kluwer Academic Publishers, 2003), pp. 277-316.

[25] V. Kotimäki, E. Räsänen, H. Hennig, and E. J. Heller, Phys. Rev. E 88, 022913 (2013).

[26] While Brownian motion and the heat diffusion equation on fractal geometries have been extensively studied in the literature $[27,28]$, the transport properties of electrons roaming on such complicated geometrical structures have comparatively received less attention. More precisely, some analytical [29, 30] and numerical [31, 32, 33, 34] studies of the conductance of electrons in Sierpinski fractals have appeared in the literature. We hasten to stress that the problem of quantum particles moving in a SC is very different from that of a quantum particle displaying a self-similar spectrum. Such problems are very well studied in physics, a paradigmatic example being that of the Hofstadter butterfly spectrum [35] displayed by an electron moving in 2D under the combined effect of a periodic potential and a perpendicular magnetic field. Finally, we are not interested in the statistical distribution of eigenvalues and the nature of the corresponding eigenstates of electrons in plane fractals, which have been studied in great detail $[36,37,38,39,40]$. Rather, our aim is to unveil fundamental de transport characteristics, which can be measured in artificially fabricated SCs with current technology.

[27] S. Havlin and D. Ben-Avraham, Adv. Phys. 36, 695 (1987); M. B. Isichenko, Rev. Mod. Phys. 64, 961 (1992).

[28] S. Kusuoka, Lecture on Diffusion Processes on Nested Fractals, Lecture Notes in Mathematics No. 1567 (Springer, Berlin, 1993), p. 39.

[29] A. Chakrabarti, J. Phys.: Condens. Matter 8, 10951 (1996).

[30] C. W. Groth, J. Tworzydło, and C. W. J. Beenakker, Phys. Rev. Lett. 100, 176804 (2008).

[31] Y. Liu, Z. Hou, P. M. Hui, and W. Sritrakool, Phys. Rev. B 60, 13444 (1999).

[32] Z. Lin, Y. Cao, Y. Liu, and P. M. Hui, Phys. Rev. B 66, 045311 (2002).

[33] S. Jana, A. Chakrabarti, and S. Chattopadhyay, Physica B 405, 3735 (2010).

[34] Z.-G. Song, Y.-Y. Zhang, and S.-S. Li, Appl. Phys. Lett. 104, 233106 (2014).

[35] D. R. Hofstadter, Phys. Rev. B 14, 2239 (1976).

[36] R. Rammal and G. Toulouse, Phys. Rev. Lett. 49, 1194 (1982).

[37] E. Domany, S. Alexander, D. Bensimon, and L. P. Kadanoff, Phys. Rev. B 28, 3110 (1983).

[38] R. Rammal, Phys. Rev. B 28, 4871(R) (1983).

[39] X. R. Wang, Phys. Rev. B 51, 9310 (1995).

[40] A. Hernando, M. Šulc, and J. Vaníček, arXiv:1503.07741.

[41] C. W. Groth, M. Wimmer, A. R. Akhmerov, and X. Waintal, New J. Phys. 16, 063065 (2014).

[42] T. Vicsek, J. Phys. A 16, L647 (1983).

[43] Y. Gefen, A. Aharony, and B. B. Mandelbrot, J. Phys. A 17, 1277 (1984). 